\title{
O USO DO TECHNOLOGY ROADMAPPING PARA A DELIMITAÇÃO DE PROPOSTAS DE PROJETOS EM UM NÚCLEO DE PESQUISA NA ÁREA DA SAÚDE
}

Jussara Queiroz de Carvalho (jussaraqc@gmail.com) - Programa de Pós-graduação em Biotecnologia Medicina Regenerativa e Química Medicinal - Universidade de Araraquara UNIARA.

Creusa Sayuri Tahara Amaral (c.sayuri.tahara@gmail.com) - Programa de Pós-graduação em Biotecnologia Medicina Regenerativa e Química Medicinal - Universidade de Araraquara UNIARA.

\section{RESUMO}

O uso do Technology Roadmapping (TRM) está difundido pelas empresas, como pode ser observado em trabalhos clássicos como em Phaal et al. (2004), mas de acordo com Souza (2010) a aplicação em pequenas empresas, startups e Núcleos de pesquisa acadêmicos sua difusão é mais recente, e há poucos relatos na área da saúde. Desde contexto surgiu a lacuna que este trabalho buscou atender. Assim, o objetivo deste artigo é apresentar os desafios de uma aplicação do método TRM para apoiar o desenvolvimento de novos projetos sobre um tema da área da saúde para o grupo de pesquisa Quimmera, do programa de pós-graduação em Biotecnologia em Medicina Regenerativa e Química Medicinal da Universidade de Araraquara - UNIARA. O trabalho utilizou o estudo de caso para a aplicação do método TRM no grupo de pesquisa, adotando a abordagem T-Plan, proposta por Phaal et al. (2004). O T-Plan é implementado a partir da realização de quatro workshops. Os workshops foram realizados com a participação dos membros do grupo de pesquisa, além de especialistas da área, como médicos e empresários. O maior desafio na aplicação do TRM foi adaptar o método para que o foco final fosse a proposta de projetos e não um produto. Isso foi possível a partir da alteração das camadas do mapa e das dinâmicas dos workshops. O TRM mostrou-se relevante para o grupo, já que o planejamento permitiu maior integração de suas competências, com uma visão focada na meta estabelecida para a elaboração de novos projetos de pesquisa. Os resultados do TRM foram mais qualitativos do que quantitativos, mas impactaram positivamente na organização do grupo de pesquisa, pela incorporação da visão de mercado, dos concorrentes e das competências, que não eram considerados na elaboração de novos projetos. No final do TRM foi aplicado um questionário para avaliar a percepção dos participantes em relação aos seus benefícios. O TRM mostrou aos pesquisadores do grupo as direções que os novos projetos devem seguir, para que em um horizonte de médio prazo sejam alcançados resultados que possam ser integrados a uma proposta de produto.

Palavras-Chave: Technology Roadmapping; Núcleos de pesquisa; Saúde

Área: Ferramentas e métodos de desenvolvimento de produtos e serviços

\section{INTRODUÇÃO}

A aplicação do método TRM tem mostrado ser significativamente positiva na busca por desenvolvimento tecnológico e no planejamento estratégico de tecnologias para as empresas. Enquanto algumas empresas aplicam o método TRM para casos específicos, outras o utilizam como parte das estratégias e processos de planejamento, constatando dessa forma quão flexível pode ser o método. O TRM pode ser um fator integrador entre processos distintos de uma organização. Integrando as necessidades e demandas externas e as competências institucionais para o desenvolvimento de projetos que venham a atender demandas, em consonância com as diretrizes estratégicas da empresa, (MERQUIOR, 2007). 
A literatura sobre TRM apresenta diversos casos de aplicação em empresas, tanto de grande porte quanto de pequeno porte, mas sobre aplicações em grupos de pesquisa e instituições de ensino são poucos casos relatados, como descrevem Carvalho et al. (2013).

Sherman et al. (2000) tem afirmado que, por um lado o conhecimento originado dentro das universidades e centros de pesquisa levam ao desenvolvimento de novas tecnologias, por outro lado, as pesquisas científicas podem ser potencializadas pelas necessidades do mercado. Este contex to sugere uma questão: Como o método TRM pode contribuir para o planejamento estratégico de novos projetos em um grupo de pesquisa? Este artigo tem como objetivo contribuir na investigação dessa questão, apresentando uma aplicação do método TRM, para o caso de um núcleo de pesquisa, de uma instituição de ensino, avaliando as necessidades de adaptação do método e quais os benefícios para a capacidade de inovação tecnológica do grupo de pesquisa. Este artigo é resultado de um projeto de pesquisa desenvolvido por Carvalho (2017).

\section{METODOLOGIA}

A metodologia adotada para a realização do trabalho tem natureza qualitativa e exploratória e utilizou-se do procedimento de estudo de caso. Segundo Gil (2002), a metodologia de natureza qualitativa possui aspecto não mensurável, baseado em percepções. Para Voss et al. (2002), o estudo de caso se caracteriza quando questões do tipo "por que" e "como" podem ser respondidas a partir da compreensão da natureza e da complexidade do fenômeno observado. De acordo com Yin (2001), há três princípios para a coleta de dados, que podem elevar a qualidade do estudo de caso:

a) Utilizar várias fontes de evidência - buscar uma ampla variedade de fontes: entrevistas, observações, provas documentais de forma que o estudo seja o conjunto da análise dessas informações e não de dados quantitativos nem qualitativos em separado;

b) Criar um banco de dados para o estudo de caso;

c) Manter o encadeamento de evidências.

Protocolo para o estudo de caso pesquisa

O protocolo de desenvolvimento do estudo de caso é um documento que serve como um roteiro facilitador para a etapa de coleta de dados. $\mathrm{O}$ protocolo abrange desde o instrumento de coleta de dados e toda a conduta a ser seguida pelo pesquisador durante o estudo de caso (YIN, 2001). Constitui-se em um elemento para mostrar a confiabilidade da pesquisa, ou seja, garante que os resultados da investigação podem ser assemelhados aos resultados da replicação do estudo de caso, ou mesmo de outro caso em condições equivalentes, orientado pelo mesmo protocolo.

O protocolo adotado para esta pesquisa foi organizado de acordo com as atividades e procedimentos que se apresentam a seguir:

a) Revisão dos principais artigos e publicações sobre o assunto abordado na pesquisa: palavras-chaves - TRM, Planejamento de tecnologia. Na pesquisa foram utilizadas as bases: Portal Capes, Scielo, Science Direct, Google Acadêmico;

b) Definição da unidade caso. Neste trabalho o caso selecionado foi o grupo de pesquisa em Medicina regenerativa e Química Medicinal - QUIMMERA, da UNIARA. O grupo tem uma história recente, pois os integrantes são docentes e alunos do recémcriado programa de pós-graduação em Biotecnologia da UNIARA;

c) Planejamento dos workshops para a aplicação do método TRM: A aplicação do 
método TRM requer um planejamento para que sejam definidas a equipe que participará do processo e quais informações iniciais devem ser levantadas para orientar a aplicação do método;

d) Seleção dos envolvidos com o projeto: a equipe para a aplicação do TRM foi definida pelo critério de áreas de competência;

e) Definição do escopo do projeto: Elaborar em conjunto com a equipe e os interessados no projeto, o objetivo a ser alcançado;

f) Processo de levantamento das informações de apoio à realização do TRM;

g) Avaliação, análise, interpretação e discussão dos resultados.

\section{PROCESSOS DE APLICAÇÃO DO MÉTODO TECHNOLOGY ROADMAPPING}

O método T-Plan, desenvolvido como um guia prático para auxiliar o processo de construção do roadmap (PHAAL et al., 2001, PHAAL et al., 2004). Segundo Phaal et al. (2004), pelos resultados das aplicações do T-plan, existe evidências de o método pode ser útil para:

a) Apoiar o início dos processos do TRM;

b) Estabelecer as ligações importantes entre os recursos de tecnologia e os direcionadores do negócio;

c) Identificar lacunas importantes no mercado, produto e de inteligência tecnológica;

d) Desenvolver um roteiro tecnológico inicial;

e) Apoiar a estratégia tecnológica e as iniciativas de planejamento na empresa;

f) Apoiar a comunicação entre funções técnicas e comerciais.

g) O processo T-Plan é constituído por quatro workshops e pela construção do mapa. $\mathrm{O}$ planejamento e aplicação dos resultados também são tarefas desse processo. As etapas do processo T-plan estão descritas a seguir:

a) Planejamento do processo, em que os objetivos, os participantes do workshop são definidos e analisados as necessidades de adaptações no processo;

b) Workshop mercado, em que se analisa direcionadores, de forma a se estabelecer objetivos e metas que devem ser atendidos pelos produtos e tecnologias;

c) Workshop produto, em que as características dos produtos são levantadas, analisadas em relação ao seu desempenho;

d) Workshop de tecnologia, em que as características da tecnologia são levantadas, analisadas em relação ao seu desempenho;

e) Implementação dos resultados: nesta fase a arquitetura é preenchida com as informações obtidas nos workshops e os resultados são divulgados e disponibilizados à empresa.

\section{DESCRIÇÃO DO CASO}

Os roadmaps elaborados para a área de ciência e tecnologia buscam delinear o futuro do desenvolvimento científico e tecnológico. No âmbito acadêmico, a identificação das aplicações atuais da tecnologia e seus potenciais desafios são estratégicos para direcionar novos projetos de pesquisa, estabelecendo as tendências e as potenciais descontinuidades. Esta foi a motivação para a seleção do estudo de caso deste trabalho, que é o grupo de pesquisa denominado Quimmera, cadastrado no grupo de pesquisa do CNPq, da Universidade de Araraquara - Uniara, do interior do Estado de São Paulo. 
O grupo de pesquisa Quimera foi criado em 2008 e desenvolve pesquisas na área de biotecnologia, nas linhas de pesquisa Química Medicinal e Medicina Regenerativa. A criação do grupo foi fundamentada em ações específicas que foram desenvolvidas pelos pesquisadores da Uniara em parceria com pesquisadores da Unicamp, USP, Unesp e UFSCar.

O grupo Quimmera tem como objetivos desenvolver ações integradas nas áreas de Química, Biologia, Medicina, Fisioterapia, Biomedicina, Farmácia, Odontologia e Engenharia de Produção, para o desenvolvimento de projetos focados em medicina regenerativa e química medicinal.

\subsection{Aplicação do TRM para planejamento de novas tecnologias em Reparo Ósseo}

A aplicação do TRM para o caso do grupo Quimera foi organizada em cinco etapas:

a) Definição do escopo do TRM: Dentre os diversos projetos desenvolvidos pelo grupo, o tema de pesquisa sobre reparo ósseo foi selecionado, após a realização de reuniões com os principais interessados na aplicação do método, que são os líderes do grupo. Este tema "Reparo ósseo" foi selecionado por ser abrangente e por estar associado a alguns projetos de mestrado do programa de pós-graduação, ao qual o grupo faz parte;

b) Elaboração de um mapa de competência do grupo de pesquisa: O ponto de partida para o desenvolvimento do trabalho foi a construção do mapa de competências do grupo de pesquisa, que foi elaborado a partir da consulta dos dados descritos no currículo Lattes de cada pesquisador do grupo, além de entrevistas e consultas diretas aos pesquisadores. O objetivo esperado do mapa de competência foi de fortalecer e potencializar a sinergia do grupo pela integração de suas competências. O mapa de competência foi um importante instrumento, pois por meio dele foi possível identificar as competências e qualificação dos integrantes do grupo de pesquisa, que refletem diretamente na capacidade do grupo para o desenvolvimento de novos projetos. A análise do mapa de competência pode mostrar necessidades de parcerias e associações para cobrir lacunas ou deficiências em capacitação do grupo;

c) Organização dos dados e informações: O processo de coleta de dados e a preparação das informações para a aplicação da metodologia não é uma atividade trivial, já que devem ser consultadas informações de áreas de conhecimento específicas, de várias fontes, como base de patentes, base de artigos acadêmicos, entrevistas com especialistas, profissionais que atuam na área, além de bases do ministério da saúde, etc. Neste trabalho foram consultadas as seguintes bases: PatentesOnline, Espacenet, USPTO, Google patentes e Periódicos capes. As entrevistas foram realizadas com médicos ortopedistas, com o apoio de um roteiro para organizar a sequência de perguntas, focadas no tema sobre reparo ósseo, que permitiram o levantamento de informações que caracterizaram as expectativas dos profissionais que utilizam os produtos disponíveis para o reparo ósseo. As entrevistas foram realizadas na fase inicial da aplicação do método e permitiu que o processo de busca de informações fosse refinado, melhorando as especificações sobre o mercado e também sobre os principais requisitos que o produto deveria ter, além de identificar os principais produtos utilizados nos procedimentos cirúrgicos. Assim, nesta etapa do processo levantamento de dados, foram consolidadas as informações sobre os principais produtos disponíveis no mercado para reparo ósseo;

d) Aplicação do T-Plan: A aplicação do Technology Roadmapping com o método T-Plan ocorre com a realização de 4 workshops, como já mencionado. Para a realização desses workshops houve a participação dos pesquisadores do grupo QUIMMERA, alunos de pós-graduação e profissionais da área (médicos e empresas). A partir de 
reuniões iniciais com os responsáveis pelo grupo de pesquisa foram definidas as informações que direcionaram o objetivo do TRM. Em cada workshop, três pessoas chave trabalharam em conjunto para planejar e dirigir a reunião: o facilitador, o colaborador e o especialista. O facilitador foi representado por um docente do programa de pós-graduação. O colaboradora foi representado por um aluno do programa, cuja função estendeu-se desde o levantamento das informações para a base de dados do projeto, como também os contatos com os participantes externos ao grupo. A participação do especialista, ou seja, um pesquisador sênior na área de TRM foi fundamental para a consolidação das informações obtidas no workshop e também para planejar e organizar o workshop subsequente. A partir de reuniões com os principais envolvidos com o processo, o escopo do TRM foi especificado. Alterações foram realizadas ao longo da aplicação do TRM, pela necessidade de adaptações ao caso. O levantamento de informações ocorreu ao longo de todo o processo de aplicação do método, considerando esta uma fase importante, pois foi a base para a elaboração do mapa;

e) Avaliação dos resultados: Podemos apontar como um dos benefícios da aplicação do TRM no grupo de pesquisa Quimmera foi a visão estratégica do processo de desenvolvimento de projetos, que em geral os pesquisadores não dispõem. O processo de elaboração do mapa para o planejamento da tecnologia realizado pelo grupo, forneceu essa visão.

\section{RESULTADOS}

A aplicação do Technology Roadmapping com o método T-Plan iniciou-se na etapa de planejamento, mas uma parte importante desse processo acontece com a realização dos workshops. Para a realização desses workshops houve a participação dos pesquisadores do grupo QUIMMERA, alunos de pós-graduação e profissionais da área (médicos e empresas), como descrevemos nas subseções seguintes.

\subsection{Workshop - Mercado / Produto}

O I workshop foi iniciado com uma apresentação do mapa de competências e uma explicação sobre os conceitos do TRM. Neste workshop foi possível reunir somente os pesquisadores dos grupos de pesquisa Quimmera e do grupo BioPolMat, que tem grande interação com o tema proposto para a aplicação do TRM. As discussões foram conduzidas com base em um roteiro de perguntas, que propunham esclarecer aspectos importantes do mercado, do produto, sobre os principais desafios de pesquisa na área de reparo ósseo e as dificuldades e barreiras enfrentadas pelo grupo, tanto tecnológicas como de competências para superar os problemas dessa área. O workshop foi conduzido por um facilitador, que foi apoiado por um pósgraduando do programa. As questões debatidas na reunião foram:

a) Quais produtos poderiam ser desenvolvidos?

b) Qual o desempenho esperado desse produto?

c) Quais as principais lacunas e limitações sobre reparo ósseo?

d) Quais as principais técnicas estão sendo utilizadas sobre reparo ósseo?

e) Quais as oportunidades?

f) Quais são as fraquezas do grupo nessa área?

g) Quais as vantagens do grupo nessa área?

Uma das informações refinadas após o I workshop foi sobre o mercado, cuja definição não foi elementar, já que o resultado do TRM, não atenderia diretamente um específico mercado. 
Assim, o que foi definido foram os principais stakeholders do projeto, que influenciariam as direções da aplicação do TRM: médicos ortopedistas e dentistas.

\subsection{Workshop - Produto / Tecnologia}

No II workshop foram apresentados os principais produtos disponíveis no mercado para reparo ósseo, as tecnologias existentes que apoiam o produto e algumas patentes importantes. Com base nestas informações buscou-se delinear as características que o novo produto deveria apresentar para ser inovador e atrativo para o mercado, mantendo os princípios básicos de ser osteocondutor e osteoindutor, características consideradas fundamentais no produto, além de viável economicamente e confiável para o paciente.

Neste workshop chegou-se a um "conceito" de produto, que seria formado por uma combinação de um scaffold (com as melhores propriedades, por exemplo ser bioativo), que deveria ser acrescido de um fator de crescimento e células (do próprio paciente). Esse modelo agruparia as características propostas para o produto e permitiria novas possibilidades de pesquisa, como a ação de novos biomateriais, como o biovidro e a proteína BMP, que estão na fronteira do conhecimento na área de medicina regenerativa.

O resultado deste workshop, na verdade não foi a definição de um produto, foi a caracterização de uma proposta de produto, que uniu as linhas de pesquisa do grupo, integrando diversos projetos com o mesmo objetivo e a interação das competências dos docentes e também de seus parceiros.

\subsection{Workshop - Validação}

O workshop teve como objetivo validar as informações levantadas e consolidadas no mapa preliminar elaborado e delinear o plano de ações. Um projeto temático resultou como proposta final do planejamento, que seria o foco de integração entre todos os esforços de pesquisa, de acordo com as áreas de competência.

\subsection{Avaliação}

A partir dos dados coletados e nas decisões tomadas em cada workshop, o mapa do TRM foi elaborado e apresentado aos envolvidos. O mapa gerado, figura 1, tornou-se uma fonte de consulta para todos os envolvidos no processo, como um documento que representa e registra os acordos estabelecidos no processo. As atualizações no mapa devem ser realizadas sempre que novas decisões sejam tomadas, à medida que o plano de ações sejam realizados ou surgirem novos elementos, que ocasionem mudanças nos caminhos, para que o mapa continue a ser um instrumento para orientação das decisões do grupo em relação às pesquisas realizadas. As bases de patentes permitiram o levantamento sobre as novas tecnologia e métodos que serão utilizados no desenvolvimento de novos produtos para reparo ósseo, fornecendo uma base de orientação para novos projetos.

Ao término do processo de aplicação do TRM, um questionário foi entregue aos participantes dos workshops para avaliar os benefícios da aplicação do método TRM. Nesta avaliação procurou-se entender como o TRM foi compreendido pelos participantes e qual a percepção dos benefícios decorrentes do TRM para o grupo. $\mathrm{O}$ questionário foi respondido individualmente por cinco pesquisadores do grupo Quimmera. Os principais benefícios percebidos pelos participantes, ilustrado na figura 2, foram:

a) Revelou lacunas, desafios e incertezas em relação a tecnologia e produto;

b) Houve fortalecimento e estruturação do processo de planejamento no médio e longo prazo; 
c) Auxiliou na definição das prioridades para o desenvolvimentode novos projetos;

d) Houve alteração nos requisitos para o desenvolvimento de um novo projeto, como por exemplo incluir o mercado, como fator de relevância do tema.

A segunda parte do questionário buscou avaliar o processo de aplicação do TRM. Os mesmos cinco pesquisadores do grupo Quimera, que responderam a primeira parte do questionário, também responderam a segunda parte. Dentre eles três participantes já conheciam o método TRM. Em relação à questão referente ao ponto mais relevante na aplicação do método, três pesquisadores se referiram à aquisição de informações e dois pesquisadores responderam sobre a integração entre pesquisadores, suas competências e suas linhas de pesquisa. Sobre falhas e lacunas no desenvolvimento do método, três pesquisadores afirmaram não ter conhecimento para avaliar. Um pesquisador julgou não ter havido falhas, já para outro pesquisador faltou a apresentação de casos em que o TRM tivesse sido aplicado, evidenciando a necessidade de detalhamento na apresentação do método. Apesar de ser o relato de apenas um participante, este fato tem relevância, pois explicaria alguns problemas de continuidade na aplicação do método, que inclusive teve etapas agrupadas, justamente para tornar o processo de mapeamento mais concreto.

\section{CONCLUSÕES}

Novos critérios das agências de fomentos e da própria sociedade para um melhor aproveitamento dos recursos financeiros aplicados em pesquisas científicas tem motivado as universidades, e as instituições de pesquisa a alocarem seus recursos de modo mais planejado e para isso tem se tornado imperativo a elaboração de estratégias de pesquisas que considerem também os interesses da sociedade (SCHWARTZMAN, (2008) apud SOUZA e ZAMBALDE.

Este trabalho buscou atender essa lacuna pela aplicação do método Technology Roadmapping (TRM), a partir da metodologia T-Plan (PHAAL et al., 2001), em um grupo de pesquisa acadêmico. Explorar a dinâmica da inovação em grupos de pesquisa de forma planejada e estruturada, permite que os estudos científicos tenham mais chances para gerar inovações e facilitar o acesso a fontes de investimentos no médio e longo prazo. A prospecção tecnológica, um resultado observado com o método TRM, pode auxiliar, pesquisadores na busca por uma visão compartilhada das demandas mais relevantes e também dos campos de estudos mais promissores para o desenvolvimento de inovações, de forma a estabelecer prioridades e articular esforços em torno de projetos que tenham impacto no conhecimento e também sejam de interesse da sociedade (ZACKIEWICZ et al., 2005; SILVA et al.,2005). Uma adaptação foi realizada na proposta de Phaal et al. (2001), em que se utilizou de três workshops para explorar as informações sobre o mercado, produto e tecnologia, sendo que a camada de tecnologia foi desdobrada em: métodos, patentes, artigos, competências e recursos, como pode ser visto na figura 1 . Também nesta aplicação incluímos a camada de projetos, para representar, de certo modo, os resultados do processo. O desdobramento da camada de tecnologia, permitiu uma visão detalhada de todos os aspectos que o grupo deveria avaliar para o desenvolvimento de novos projetos. 
Figura 1 - Roadmap para o caso de reparo ósseo do Grupo Quimmera. Fonte: Carvalho (2017)

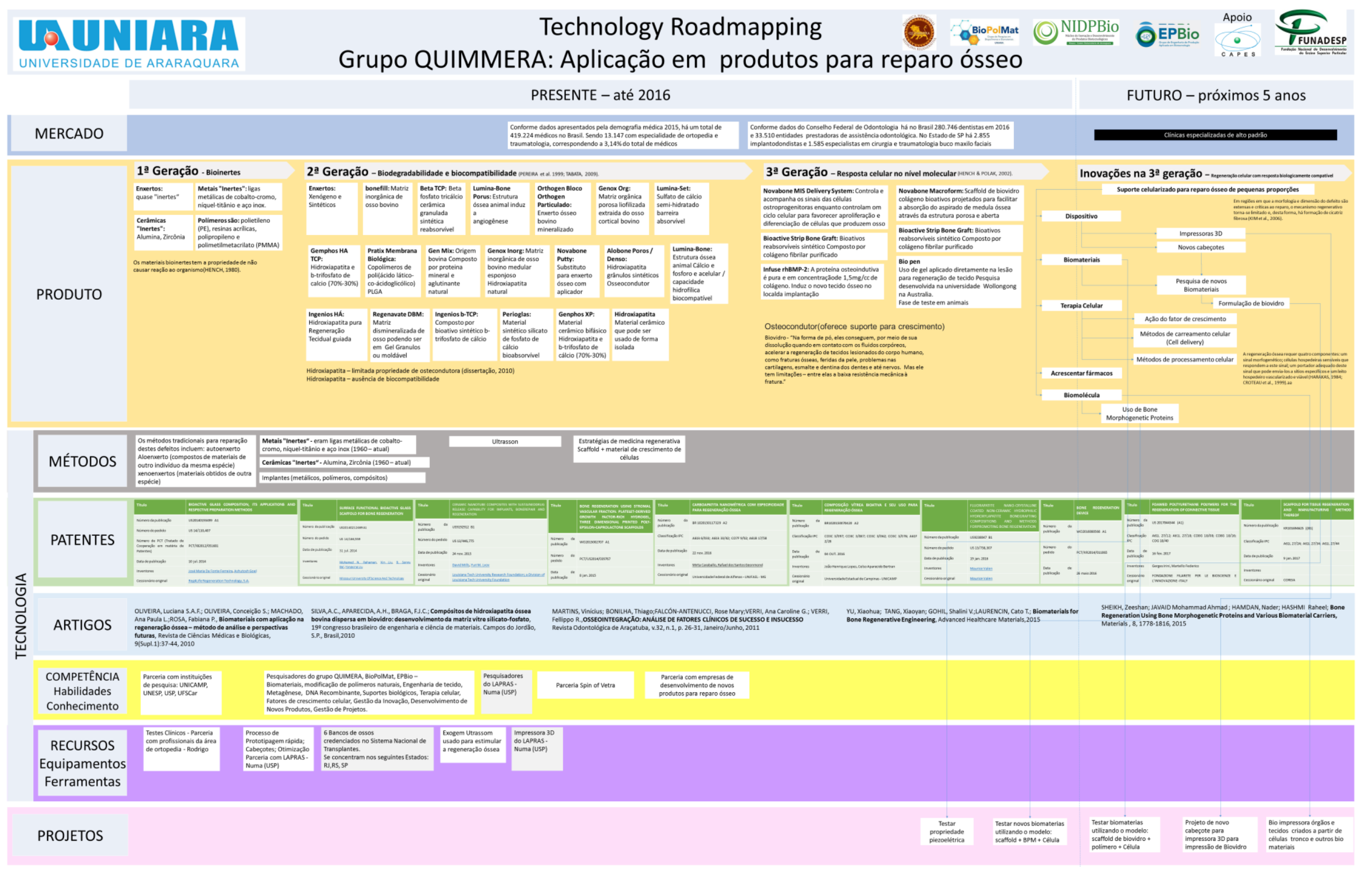


04 e 05 de setembro de 2017 - Escola Politécnica da Universidade de São Paulo, São Paulo, SP

Figura 1 - Médias ordenadas para a avaliação da percepção dos benefícios do método TRM. Fonte: Carvalho (2017)

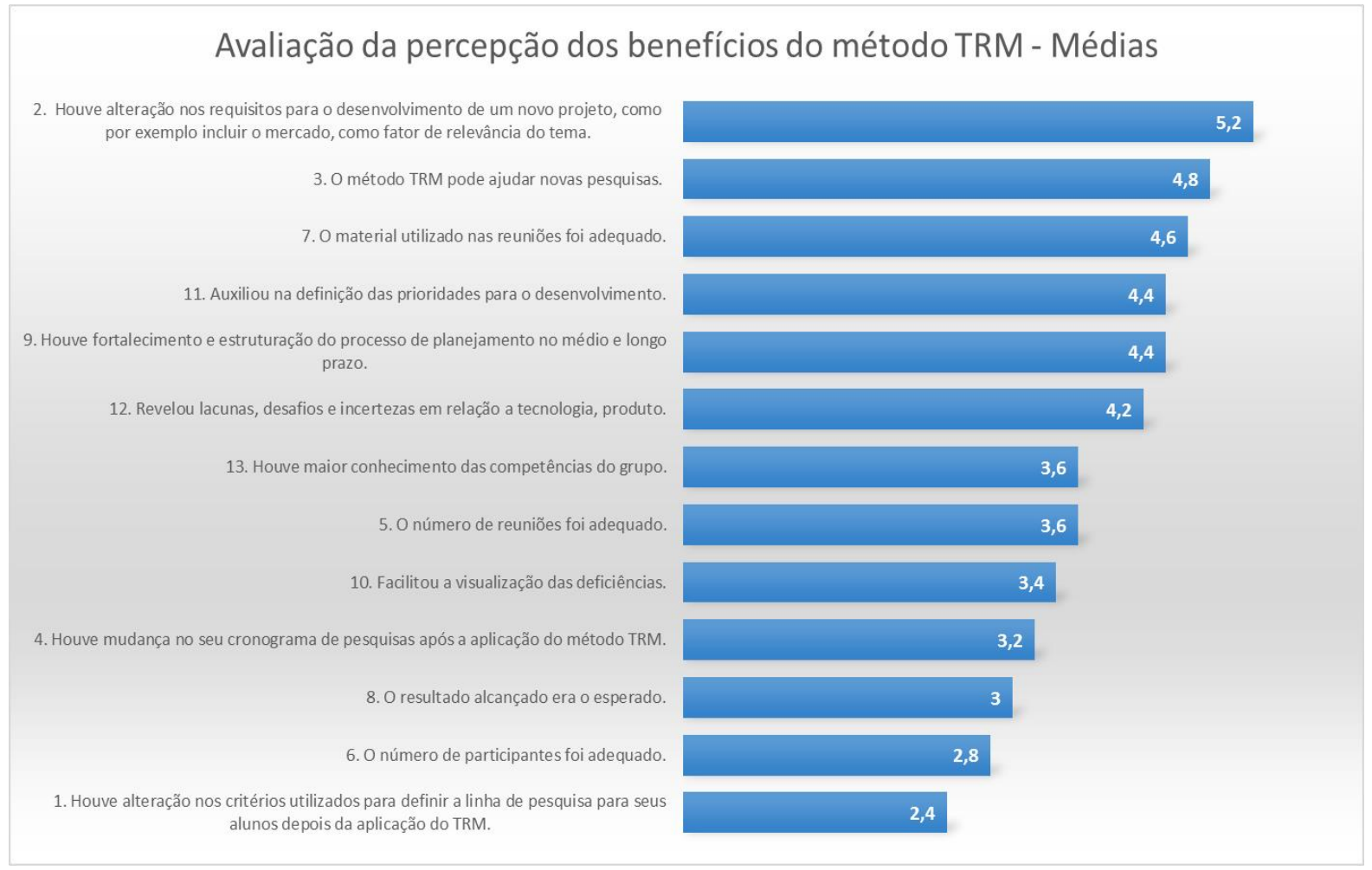

Dentre as principais limitações do estudo, destaca-se a o caráter restritivo da aplicação, já que para identificarmos melhores práticas, deveríamos estender o estudo para múltiplos casos. Uma dificuldade na aplicação foi atingir um nível uniforme de compreensão sobre os conceitos básicos do método, para que os pesquisadores pudessem entender sobre os resultados que o método proporcionaria ao grupo e os reais benefícios às pesquisas. Perceber a importância do mapa de competências gerou benefícios ao grupo, que resultou em um projeto de elaboração do planejamento estratégico para o programa de pós-graduação. Esse conhecimento também permitiu maior sinergia do grupo de pesquisa, em relação a novas oportunidades de interação. Outra questão importante é que este trabalho pode levar para a área da saúde casos de aplicação de métodos tradicionais da engenharia de produção, que são poucos divulgados no contexto da saúde, mas têm se mostrado contribuir para o estabelecimento de melhores práticas na gestão de processos e desenvolvimento de produtos. Assim, pôde-se preparar o grupo de pesquisa para a realização de estudos estruturados e prospectivos, visando não somente as publicações científicas, mas também aspectos práticos relacionados ao mercado, contribuindo para potencializar as competências do grupo de pesquisa, iniciando o processo de institucionalização do planejamento estratégico das pesquisas, valorizando as atividades de gestão das informações geradas por pesquisas científica, uma das principais características do TRM.

A aplicação do TRM no grupo de pesquisa Quimmera contribuiu para introduzir as práticas de planejamento estratégico para as pesquisas científicas, que devem ser capazes de contribuir para futuras iniciativas empreendedoras e transferências de tecnologias para o mercado e para a sociedade. 


\section{REFERÊNCIAS}

CARVALHO, J. Q., Aplicação do Technology Roadmapping no desenvolvimento de produtos biotecnológicos em um núcleo de pesquisa: um estudo de caso. 2017, 82p. Dissertação (Mestrado), Programa de Pós-Graduação em Biotecnologia Medicina Regenerativa e Química Medicinal, Universidade de Araraquara - UNIARA, SP, 2017.

CARVALHO, M. M.; FLEURY, A.; LOPES, A. P., An overview of the literature on technology roadmapping (TRM): Contributions and trends, Technological Forecasting \& Social Change, v. 80, p. 1418-1437, 2013.

GIL, A.; C. Como elaborar projetos de pesquisa. São Paulo: Atlas 4 ed, 2002.

MERQUIOR, D. M. Gestão de Inovações e Tecnologia: Roadmap de tecnologia. PADECEME, Rio de Janeiro, n.16, p.34-44, 2007.

PHAAL, R.; FARRUKH, C. J. P.; PROBERT, D. R. Tecnology roadmapping-A planning framework for evolution and revolution, Technological Forecasting and Social Change, v. 71, n. 1-2, p. 5-26, 2004.

PHAAL, Robert; FARRUKH, Clare; PROBERT, David. T-plan: fast start technology roadmapping: a practical guide for supporting technology and product planning. University of Cambridge, 2001.

SHERMAN, J. Daniel, SOUDER, William E., and JENSSEN, Svenn A. SLACK, N.; et al. Administração da Produção, São Paulo: ed Atlas, p. 74,232, 1999.

SILVA, A. M. A. C. da; ZACKIEWICK, M.; BONACELLI, M. B. M.; Indicadores para monitoramento de ciência e tecnologia e apoio à decisão, 2005, Científico Internacional, Seminário Latino-Iberoamericano de Gestión Tecnológica - ALTEC'2005, v.1, p.1-16, 2005.

SOUZA, D. L.; ZAMBALDE, A. L.; Prospecção tecnológica em grupos de pesquisa acadêmicos: uma proposta metodológica a partir do Technology Roadmapping (TRM), Revista Espacios, v. 37, n.32, 2016.

SOUZA, M. C. Método de mapeamento de tecnologia considerando a estratégia technology push e adoção de parcerias, 2010. 201 f. Tese (Doutorado-Programa de Pós-Graduação em Engenharia de Produção e área de Concentração Processos e Gestão de Operações) - Escola de Engenharia de São Carlos, Universidade de São Paulo, 2010.

Schwartzman, S. (2008). The Leading Latin American Universities and Their Contribution to sustainable development in the region. In: Schwartzman, S. (Org.). University and Development in Latin America: Successful Experiences of Research Centers. Institute of Labor and Society Studies, Rio de Janeiro: Sense Publishers, pp. 5-20 apud SOUZA, D. L.; ZAMBALDE, A. L.; Prospecção tecnológica em grupos de pesquisa acadêmicos: uma proposta metodológica a partir do Technology Roadmapping (TRM), Revista Espacios, v. 37, n.32, 2016.

VOSS, C.; TSIKRIKTSIS, N.; FROHLICH, M. Case research in operations management, International Journal of Operations \& Production Management, v. 22, n. 2, p. 195 - 219, 2002.

YIN, R, K. Estudo de caso: planejamento e método. $2^{\text {a }}$ ed. Porto Alegre ,-RS: Bookman, 2001.

ZACKIEWICK, M.; BONACELLI, M. B. M.; SALLES FILHO, S. L. M.; Estudos prospectivos e a organização de sistemas de inovação no Brasil, 05/2005, São Paulo em Perspectiva, v. 19, p.115-121, 2005. 